\title{
Article \\ Full-Order Terminal Sliding-Mode Control of Brushless Doubly Fed Induction Generator for Ship Microgrids
}

\author{
Minghao Zhou ${ }^{1}$, Hongyu Su ${ }^{1}$, Yi Liu ${ }^{2,3}$, William Cai ${ }^{1, *}$, Wei Xu ${ }^{2}$ and Dong Wang ${ }^{1}$ \\ 1 School of Electrical and Electronic Engineering, Harbin University of Science and Technology, \\ Harbin 150001, China; zhouminghao@aliyun.com (M.Z.); suzuhys@163.com (H.S.); \\ wdong7654@163.com (D.W.) \\ 2 School of Electrical and Electronic Engineering, Huazhong University of Science and Technology, \\ Wuhan 430074, China; liuyi82@hust.edu.cn (Y.L.); weixu@hust.edu.cn (W.X.) \\ 3 State Key Laboratory of Advanced Electromagnetic Engineering and Technology, Wuhan 430074, China \\ * Correspondence: william_cai88@163.com
}

Citation: Zhou, M.; Su, H.; Liu, Y.; Cai, W.; Xu, W.; Wang, D. Full-Order Terminal Sliding-Mode Control of Brushless Doubly Fed Induction Generator for Ship Microgrids. Energies 2021, 14, 7302. https:// doi.org/10.3390/en14217302

Academic Editors: Jingang Lai and Armando Pires

Received: 9 September 2021

Accepted: 13 October 2021

Published: 4 November 2021

Publisher's Note: MDPI stays neutral with regard to jurisdictional claims in published maps and institutional affiliations.

Copyright: (c) 2021 by the authors. Licensee MDPI, Basel, Switzerland. This article is an open access article distributed under the terms and conditions of the Creative Commons Attribution (CC BY) license (https:/ / creativecommons.org/licenses/by/ $4.0 /)$.

\begin{abstract}
This paper proposes a full-order terminal sliding-mode (FOTSM) control scheme for brushless doubly fed induction generator (BDFIG)-based islanded microgrids. To deal with mismatched uncertainties in the BDFIG system, virtual control technique-based full-order sliding-mode control is applied to stabilize the amplitude and frequency of terminal voltage. In the current loops, two full-order terminal sliding-mode controllers are designed to make sure that the current tracking errors can reach their equilibrium points in finite time. It is demonstrated by the comprehensive simulations that the proposed method can significantly improve the tracking accuracy, the rapidness, and the robustness to the uncertainties of the BDFIG control system and can enhance the output voltage quality. Furthermore, an experimental study of the proposed control method for BDFIG-based islanded microgrids would be another important future work.
\end{abstract}

Keywords: brushless doubly fed induction generator (BDFIG); islanded microgrids; sliding-mode control (SMC); terminal sliding-mode; mismatched uncertainties

\section{Introduction}

The brushless doubly fed induction generator (BDFIG) has demonstrated promising prospects in the energy-saving performance of microgrids, such as applications in wind power systems, ship shaft power generation systems, etc. Among the members of the induction motor (IM) family, the BDFIG has no brushes and slip rings, which is the main difference from the traditional doubly fed induction generator (DFIG) [1-3]. The BDFIG not only inherits the merits of the DFIG but also shows higher reliability and longer work lifetime. Therefore, it has been regarded as a feasible alternative [4,5].

The BDFIG consists of a rotor and two stator windings with different pole pairs. The indirect electromagnetic coupling effect between the two stator is generated via a specially designed squirrel-cage type rotor without the rotor voltage [6,7]. The BDFIG-based system for a ship microgrid is depicted in Figure 1. The BDFIG is connected to the main engine of the ship, the power winding (PW) is directly joined to the grid, while the control winding $(\mathrm{CW})$ is supplied by a back-to-back converter achieving the bidirectional power flow [8]. The PW-side converter maintains the stability of the DC-bus voltage, and the CW-side converter regulates the frequency variable exciting current.

The control strategies proposed for BDFIGs include the typical vector control (VC) [9]; the direct torque control (DTC) [10]; and some others such as the phase angle control [11], indirect stator quantity control [12], etc. The DTC has advantages such as rapidness and robustness under operating conditions, but large torque/power ripples and currentwaveform distortions will degrade the quality of the output power [13]. While in the VC system, the current harmonic spectra are better than those under the DTC. However, 
the common controllers are sensitive to inner parameter perturbation and external disturbances. The controller design for the BDFIG-based microgrid is somewhat difficult to develop for the following two main reasons: (1) the mathematical model of the BDFIG is a class of highly nonlinear multi-input-multi-output (MIMO) systems with unavoidable uncertainties and lots of degrees of freedom; (2) the parameters of the PW, the CW, and the rotor vary with current frequency, motor temperature, and magnetic saturation effect. The existing controller design algorithms are generally heavy parameter-dependence, meanwhile common and practical proportional-integral (PI) control cannot meet the requirements of control objectives well in the precision and dynamic performance [14,15]. Therefore, it is necessary to design advanced nonlinear controllers to compensate for the influence of parameter variations in the system, so that the BDFIG-based microgrid can obtain a good operating performance in various environments and conditions.

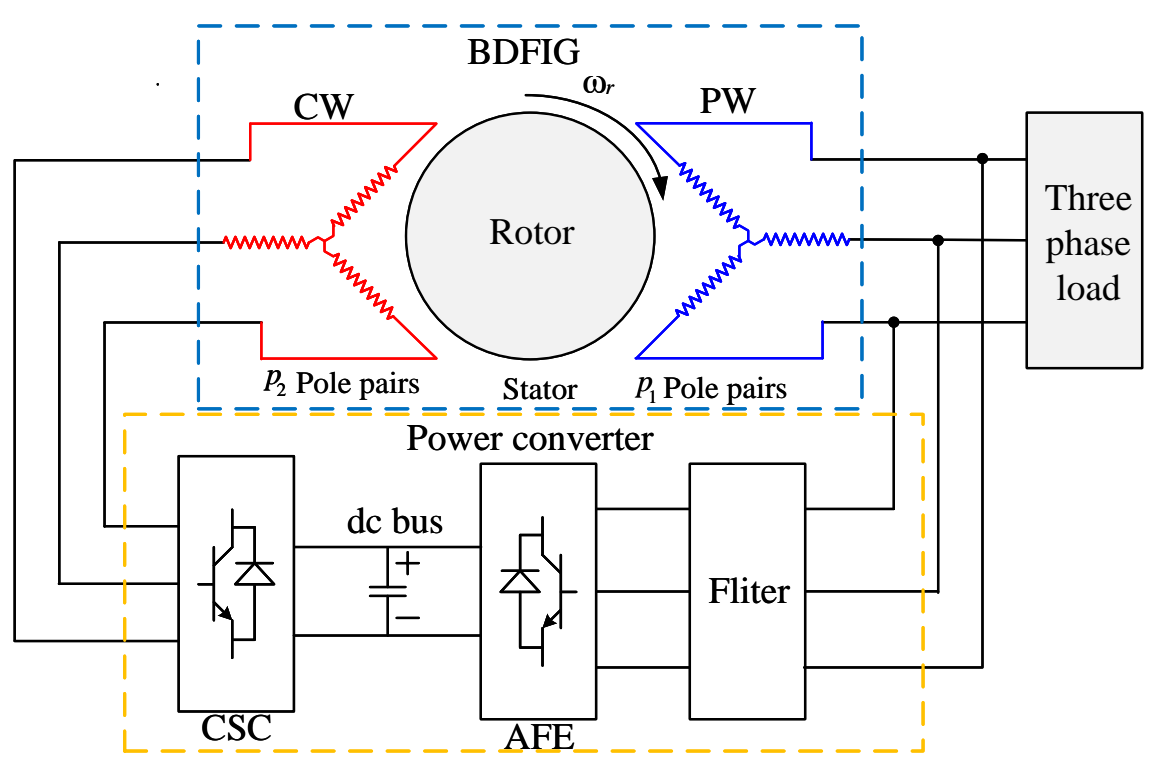

Figure 1. The structure of the BDFIG in the ship power mircogrid, where the SCS means the CW-side converter and the AFE means the active front end.

With the development of control theory, lots of control methods for DFIG systems have been proposed to implement high-performance control for the BDFIG, such as backstepping control [16], adaptive control [17], $H \infty$ control [18], fuzzy control [19], model reference adaptive control (MRAC) [20], model predictive control (MPC) [21], active disturbance rejection control (ADRC) [22-24], neural network control [25], sliding-mode control (SMC) [26], etc.

Due to the low sensitivity to parameter perturbations and the strong robustness to external disturbance, the sliding mode control (SMC) is proposed for the IM [27,28], the DFIG [29], and the BDFIGs [30]. An integral sliding-mode-based DTC scheme is proposed for DFIG-based wind-energy conversion systems in [31], and the advantages against parameter variations is illustrated. However, the convergence is not finite and undesired chattering exists. To deal with the chattering problem, an adaptive internal SMC is developed to optimize the efficiency of variable-speed wind turbines and to meet the control requirements of wind energy conversion systems under model uncertainties, which generates smoothed active power with minimized ripple [32]. However, a discrete control output with a high switching frequency is needed when applying this method. Alternatively, the second-order SMC can eliminate chattering without a discrete output [33,34]. However, the mathematical calculations of this method are complex and the implementation becomes difficult in the case of the increasing states [35]. A saturation function-based integral SMC with feed-forward terms is proposed for the BDFIG, but a quasi-sliding mode controller 
utilizing the boundary layer would decrease the control accuracy [36]. In [34], a super twisting sliding mode direct power control scheme for the BDFIGs is implemented. Although the super twisting SMC is an effective way to suppress the chattering with continuous signals, it is non-monotonically convergent to the sliding manifold and may cause a large overshoot [37].

The mathematical model of the BDFIG is a class of second-order nonlinear systems with matched and mismatched uncertainties. However, the SMC in general is regarded as an excellent method for nonlinear systems, while until now, most SMC methods depended on the so-called matched conditions [31]. The reference of $d$ - and $q$-currents designed by the traditional SMC contains high-frequency switching signals, and its derivative existing in the signals of space vector pulse width modulation (SVPWM) would lead to the singularity problem. In existing works, little attention has been paid to this issue. Therefore, to improve the accuracy rapidness and robustness to the uncertainties, advanced control methods for the BDFIGs are demanded to have a strong disturbance rejection capability for mismatched uncertainties.

The full-order terminal sliding-mode (FOTSM) is one of the most recent proposed SMC theory, which is proven to be efficient for mismatched nonlinear systems [38]. By introducing the ideal of both the full-order terminal sliding-mode (FOTSM) control algorithm and the virtual control technique, this paper proposes a novel FOTSM-based control method to stabilize the amplitude and frequency of terminal voltage under the parameter perturbation and electrical load vary. The main contributions in this article can be summarized as follows:

(1) Compared with the conventional controllers, the control precision and transient response of the amplitude and frequency of terminal voltage improved by adopting the proposed FOTSM controllers without using low pass filters or the boundary layer method.

(2) On the premise that the excellent anti-disturbance of the SMC is kept in case of uncertainties, the chattering in the conventional SMC is mitigated by the integral control law and continuous pulse width modulation (PWM) output signals can be generated.

(3) The matched and mismatched uncertainties in the BDFIG system are adequately considered and thoroughly compensated. The adaptive gain is utilized to avoid overestimating the upper bound of the uncertainties and to improve the adaptation of the BDFIG system to the uncertainties.

This paper is organized as follows: Section 2 introduces the mathematical model of the BDFIG with uncertainties. In Section 3, the voltage amplitude- and current-loop controllers based on FOTSM are designed. Section 4 gives the simulation results. Finally, the conclusions are summarized in Section 5.

\section{Preliminary}

\subsection{Dynamic Model of the BDFIG}

The dynamic mathematical model of the BDFIG in the $d-q$ coordinate with the angle frequency $\omega_{2}$ of the rotating can be expressed as

$$
\begin{gathered}
\left\{\begin{array}{l}
u_{1 d}=R_{1} i_{1 d}+s \phi_{1 d}-\omega_{2} \phi_{1 q} \\
u_{1 q}=R_{1} i_{1 q}+s \phi_{1 q}+\omega_{2} \phi_{1 d}
\end{array}\right. \\
\left\{\begin{array}{l}
u_{2 d}=R_{2} i_{2 d}+s \phi_{2 d}-\left[\omega_{2}-\left(p_{1}+p_{2}\right) \omega_{r}\right] \phi_{2 q} \\
u_{2 q}=R_{2} i_{2 q}+s \phi_{2 q}+\left[\omega_{2}-\left(p_{1}+p_{2}\right) \omega_{r}\right] \phi_{2 d}
\end{array}\right. \\
\left\{\begin{array}{l}
u_{r d}=R_{r} i_{r d}+s \phi_{r d}-\left(\omega_{2}-p_{1} \omega_{r}\right) \phi_{r q} \\
u_{r q}=R_{r} i_{r q}+s \phi_{r q}+\left(\omega_{2}-p_{1} \omega_{r}\right) \phi_{r d}
\end{array}\right. \\
\left\{\begin{array}{l}
\phi_{1 d}=L_{1} i_{1 d}+L_{1 r} i_{r d} \\
\phi_{1 q}=L_{1} i_{1 q}+L_{1 r} i_{r q}
\end{array}\right.
\end{gathered}
$$




$$
\begin{gathered}
\left\{\begin{array}{l}
\phi_{2 d}=L_{2} i_{2 d}+L_{2 r} i_{r d} \\
\phi_{2 q}=L_{2} i_{2 q}+L_{2 r} i_{r q}
\end{array}\right. \\
\left\{\begin{array}{l}
\phi_{r d}=L_{r} i_{r d}+L_{1 r} i_{1 d}+L_{2 r} i_{2 d} \\
\phi_{r q}=L_{r} i_{r q}+L_{1 r} i_{1 q}+L_{2 r} i_{2 q}
\end{array}\right.
\end{gathered}
$$

and the rotor speed of the BDFIG can be expressed as

$$
\omega_{1}+\omega_{2} \cong\left(p_{1}+p_{2}\right) \omega_{r} .
$$

Setting the rotor voltages to zero $u_{r d}=u_{r q}=0$, the voltage equation of rotor (3) can be rewritten as

$$
\left\{\begin{array}{l}
0=R_{r} i_{r d}+s \phi_{r d}-\left[\omega_{2}-p_{1} \omega_{r}\right] \phi_{r q} \\
0=R_{r} i_{r q}+s \phi_{r q}+\left[\omega_{2}-p_{1} \omega_{r}\right] \phi_{r d}
\end{array} .\right.
$$

Substituting (6) and (7) into (8) yields

$$
\begin{gathered}
i_{r d}=-\frac{\left[L_{r} s^{2}+R_{r} s+L_{r}\left(\omega_{1}-p_{2} \omega_{2}\right)^{2}\right]\left(L_{1 r} i_{1 d}+L_{2 r} i_{2 d}\right)}{\left(R_{r}+L_{r} s\right)^{2}+L_{r}^{2}\left(\omega_{1}-p_{2} \omega_{r}\right)^{2}} \\
-\frac{R_{r}\left(\omega_{1}-p_{2} \omega_{r}\right)\left(L_{1 r} i_{1 q}+L_{2 r} i_{2 q}\right)}{\left(R_{r}+L_{r} s\right)^{2}+L_{r}^{2}\left(\omega_{1}-P_{2} \omega_{r}\right)^{2}} \\
i_{r q}=\frac{\omega_{1}-p_{2} \omega_{r}}{R_{r}+L_{r} s}\left[1-\frac{L_{r}^{2} s^{2}+L_{r} R_{r} s+L_{r}^{2}\left(\omega_{1}-p_{2} \omega_{r}\right)^{2}}{\left(R_{r}+L_{r} s\right)^{2}+L_{r}^{2}\left(\omega_{1}-p_{2} \omega_{r}\right)^{2}}\right]\left(L_{1 r} i_{1 d}+L_{2 r} i_{2 d}\right) \\
-\frac{1}{R_{r}+L_{r} s}\left(L_{1 r} i_{1 q}+L_{2 r} i_{2 q}\right)\left[s+\frac{L_{r} R_{r}\left(\omega_{1}-p_{2} \omega_{r}\right)^{2}}{\left(R_{r}+L_{r} s\right)^{2}+L_{r}^{2}\left(\omega_{1}-p_{2} \omega_{r}\right)^{2}}\right] .
\end{gathered}
$$

In general, the values of $R_{r}$ are close to $L_{r}$. According to (7), the rotor speed range is limited. Hence, the $\omega_{2}$ is generally limited to $30 \%$ of $\omega_{1}$. Therefore, the first term of (9) can be rewritten as

$$
i_{r d}=-\frac{\left[s^{2}+\left(R_{r} / L_{r}\right) s+\left(\omega_{1}-p_{2} \omega_{2}\right)^{2}\right]\left(L_{1 r} i_{1 d}+L_{2 r} i_{2 d}\right)}{L_{r}\left[s^{2}+2\left(R_{r} / L_{r}\right) s+\left(\omega_{1}-p_{2} \omega_{r}\right)^{2}\right]} .
$$

Considering that the zeroes and poles of this term can cancel each other, the Equation (11) can be simplified as

$$
i_{r d}=-\frac{L_{1 r} i_{1 d}+L_{2 r} i_{r d}}{L_{r}}-\frac{R_{r}\left(L_{1 r} i_{1 q}+L_{2 r} i_{2 q}\right)}{L_{r}^{2}\left(\omega_{1}-p_{2} \omega_{r}\right)} .
$$

Similarly, Equation (10) can be simplified as

$$
i_{r q}=-\frac{L_{1 r} i_{1 q}+L_{2 r} i_{2 q}}{L_{r}} .
$$

According to (12) and (13), the voltage function of the CW (2) can be rewritten as

$$
\begin{aligned}
& \frac{\mathrm{d} i_{2 d}}{\mathrm{~d} t}=\sigma_{2} L_{2}\left(u_{2 d}-R_{2} i_{2 d}+D_{2 d}\right) \\
& \frac{\mathrm{d} i_{2 q}}{\mathrm{~d} t}=\sigma_{2} L_{2}\left(u_{2 q}-R_{2} i_{2 q}+D_{2 q}\right)
\end{aligned}
$$


where $D_{2 d}=\alpha_{1} i_{2 q}+\alpha_{2} i_{1 d}+\alpha_{3} i_{1 q}, D_{2 q}=\alpha_{4} i_{2 d}+\alpha_{5} i_{1 d}+\alpha_{6} i_{1 q}, \sigma_{2}=1-L_{2 r}^{2} /\left(L_{2} L_{r}\right)$,

$$
\begin{aligned}
& \alpha_{1}=-\frac{\omega_{1}\left(\omega_{1}-p_{2} \omega_{r}\right)\left(L_{r}^{2} L_{2}+L_{2 r}^{2} L_{r}\right)-L_{2 r}^{2} R_{r} s}{L_{r}^{2}\left(\omega_{1}-p_{2} \omega_{r}\right)}, \alpha_{2}=\frac{L_{1 r} L_{2 r} s}{L_{r}}, \\
& \alpha_{3}=-\frac{L_{1 r} L_{2 r}\left[R_{r} s+L_{r} \omega_{1}\left(\omega_{1}-p_{2} \omega_{r}\right)\right]}{L_{r}^{2}\left(\omega_{1}-p_{2} \omega_{r}\right)}, \alpha_{4}=\frac{\sigma_{2} L_{2} L_{r} \omega_{1}}{L_{1}}, \\
& \alpha_{5}=-\frac{\omega_{1} L_{1 r} L_{2 r}}{L_{r}}, \alpha_{6}=-\frac{L_{1 r} L_{2 r}\left[\omega_{1} R_{r}-L_{r}\left(\omega_{1}-p_{2} \omega_{r} s\right)\right]}{L_{r}^{2}\left(\omega_{1}-p_{2} \omega_{r}\right)} .
\end{aligned}
$$

The currents of $\mathrm{CW}$ are assumed as $i_{2 q}=0, i_{2 d}=I_{2}$. Substituing (14) and (15) into (1), the voltage of the PW can be expressed as

$$
\begin{gathered}
u_{1 d}=R_{1} i_{1 d}+\frac{\omega_{2}\left(L_{1 r}^{2}-L_{1} L_{r}\right)}{L_{r}} i_{1 q} \\
u_{1 q}=R_{1} i_{1 q}-\frac{\omega_{2}\left(L_{1 r}^{2}-L_{1} L_{r}\right)}{L_{r}} i_{1 d}-\frac{\omega_{2} L_{1 r} L_{2 r}}{L_{r}} I_{2} .
\end{gathered}
$$

The PW voltage can be calculated by

$$
\begin{aligned}
U_{1} & =\sqrt{u_{1 d}^{2}+u_{1 q}^{2}} \\
& =\left\{\left(\omega_{2} \frac{L_{1 r} L_{2 r}}{L_{r}}\right)^{2} I_{2}^{2}-\frac{2 \omega_{2} L_{1 r} L_{2 r}}{L_{r}}\left[\left(\omega_{2} L_{1}-\frac{\omega_{2} L_{1 r}^{2}}{L_{r}}\right) i_{1 d}+R_{1} i_{1 q}\right] I_{2}\right. \\
& \left.+R_{1}^{2}\left(i_{1 d}^{2}+i_{1 q}^{2}\right)+\left(\omega_{2} L_{1}-\frac{\omega_{2} L_{1 r}^{2}}{L_{r}}\right)\left(i_{1 d}^{2}+i_{1 q}^{2}\right)\right\}^{1 / 2} .
\end{aligned}
$$

For a small perturbation in the control output $\Delta I_{2}$, Equation (18) can be described by

$$
U_{1}\left(I_{2 E}+\Delta I_{2}\right)=U_{1}\left(I_{2 E}\right)+\left.\Delta I_{2} \frac{\mathrm{d} \Delta U_{1}}{\mathrm{~d} t}\right|_{I_{2}=I_{2 E}}
$$

where $I_{2 E}$ is the amplitude of a steady-state value of the CW current. Then, considering (18) and (19) yields

$$
\begin{aligned}
\Delta U_{1} & =U_{1}\left(I_{2 E}+\Delta_{I_{2}}\right)-U\left(I_{2 E}\right) \\
& =U_{1}\left(I_{2 E}+\Delta I_{2}\right)-U_{1 r e f} \\
& =K_{u} \Delta I_{2 E}
\end{aligned}
$$

where $K_{u}=\omega_{2} L_{1 r} L_{2 r}\left[\omega_{2} L_{1 r} L_{2 r} I_{2 E}+\omega_{2}\left(L_{1 r}^{2}-L_{1} L_{r}\right) i_{1 d}-R_{1} L_{1 r} i_{1 q}\right]$ is a constant in the steady-state. According to (19), the amplitude of the steady-state value of $C W$ current can be calculated as

$$
I_{2 E}=\left[\left(\beta_{1} i_{1 d}+R_{1} i_{1 q}\right)+\left(2 \beta_{1} R_{1} i_{1 d} i_{1 q}-R_{1}^{2} i_{1 d}^{2}-\beta_{1}^{2} i_{1 q}^{2}+U_{r e f}\right)^{1 / 2}\right] / \beta_{2}
$$

where $\beta_{1}=\omega_{2}\left(L_{1}-L_{1 r}^{2} / L_{r}\right), \beta_{2}=\omega_{2} L_{1 r} L_{2 r} / L_{r}$.

\subsection{Outer Loop Subsystem}

The outer-loop subsystem is the PW-voltage loop. The tracking error can be defined as $e_{U}=U_{1 \text { ref }}-U_{1}$. According to the mathematical model of the BDFIG, the voltage tracking error dynamics can be expressed by

$$
\dot{e}_{U}=\dot{U}_{r e f}-\dot{U}_{1}=-K_{u} \Delta \dot{I}_{2} .
$$


Considering the change in temperature and frequency, the variation of resistance and inductance can be represented by

$$
\left\{\begin{array}{l}
R_{i}=R_{i 0}+\Delta R_{i} \quad i=1,2, r \\
L_{j}=L_{j 0}+\Delta L_{j} \quad j=1,2, r, 1 r, 2 r
\end{array}\right.
$$

Furthermore,

$$
\left\{\begin{array}{l}
\sigma_{2}=\sigma_{20}+\Delta \sigma_{2} \\
\beta_{k}=\beta_{k 0}+\Delta \beta_{k} \quad k=1,2 \\
\alpha_{m}=\alpha_{m 0}+\Delta \alpha_{m} \quad m=1, \ldots, 9 \\
T_{1}=R_{2} \sigma_{2} L_{2}=T_{10}+\Delta T_{1} \\
T_{2}=\sigma_{2} L_{2}=T_{20}+\Delta T_{2}
\end{array}\right.
$$

The above uncertainties can be assumed to be bounded; therefore,

$$
\begin{cases}\left|\Delta R_{i}\right| \leq M_{R_{i}} & i=1,2, r \\ \left|\Delta L_{j}\right| \leq M_{L_{j}} & j=1,2, r, 1 r, 2 r\end{cases}
$$

Furthermore,

$$
\begin{cases}\left|\Delta \sigma_{2}\right| \leq M_{\sigma_{2}} & \\ \left|\Delta \beta_{k}\right| \leq M_{\beta_{k}} & k=1,2 \\ \left|\Delta \alpha_{m}\right| \leq M_{\alpha_{m}} & m=1, \ldots, 9 \\ \left|\Delta T_{n}\right| \leq M_{T_{n}} & n=1,2\end{cases}
$$

where $M_{R_{j}}, M_{L_{k}}, M_{\sigma_{2}}, M_{\beta_{1}}$, and $M_{\beta_{2}}$ are known to be positive constants.

Therefore, considering the parameter perturbation, the error dynamics in the outerloop can be described by

$$
\begin{aligned}
& \dot{e}_{U}=-K_{u} \Delta \dot{I}_{2} \\
& =-\omega_{2}\left(L_{1 r 0}+\Delta L_{1 r}\right)\left(L_{2 r 0}+\Delta L_{2 r}\right)\left[\begin{array}{l}
\omega_{2}\left(L_{1 r 0}+\Delta L_{1 r}\right)\left(L_{2 r 0}+\Delta L_{2 r}\right) I_{2 E} \\
+\omega_{2}\left(L_{1 r 0}+\Delta L_{1 r}\right)^{2} i_{1 d} \\
-\omega_{2}\left(L_{1 r 0}+\Delta L_{1 r}\right)\left(L_{r 0}+\Delta L_{r}\right) i_{1 d} \\
-\left(R_{10}+\Delta R_{1}\right)\left(L_{10}+\Delta L_{1}\right) i_{1 q}
\end{array}\right] \Delta \dot{I}_{2} .
\end{aligned}
$$

The virtual control law is defined by

$$
i_{2 d r e f}=\Delta I_{2 r e f}+I_{2 E} .
$$

The current tracking error of $i_{d}$ is defined as

$$
e_{i d}=i_{\text {dref }}-i_{d} .
$$

Thus, the tracking error dynamics (22) can be expressed as

$$
\dot{e}_{U}=-K_{u 0} \Delta \dot{I}_{2 r e f}-\Delta K_{u} \Delta \dot{I}_{2 r e f}+K_{u 0} \dot{e}_{i d}
$$

where $K_{u 0}=\omega_{2} L_{1 r 0} L_{2 r 0}\left[\omega_{2} L_{1 r 0} L_{2 r 0} I_{2 E}+\omega_{2}\left(L_{1 r 0}^{2}-L_{10} L_{r 0}\right) i_{1 d}-R_{10} L_{1 r 0} i_{1 q}\right]$ and $\Delta K_{u} \Delta \dot{I}_{2 r e f}$ is defined as the coefficient of uncertain control gain in the control system of the BDFIG.

For analyzing the lumped unmatched uncertainty $\rho_{0}$, the following uncertainties are defined as

$$
\delta_{U}=\frac{\Delta K_{u}}{K_{u 0}}=\frac{K_{u}-K_{u 0}}{K_{u 0}}
$$

which is upper-bounded by

$$
\left|\delta_{U}\right| \leq M_{\delta}<1
$$




\subsection{Inner Loop Subsystem}

According to (14) and (15), the inner-loop current subsystem can be obtained by

$$
\frac{\mathrm{d} i_{2 d q}}{\mathrm{~d} t}=\boldsymbol{F}_{0} i_{2 d q}+G\left(\boldsymbol{u}_{2 d q}+\boldsymbol{F}_{1} i_{2 d q}+\boldsymbol{F}_{2} \boldsymbol{i}_{1 d q}\right)
$$

where $\boldsymbol{i}_{2 d q}=\left[i_{2 d}, i_{2 q}\right]^{T}, \boldsymbol{u}_{2 d q}=\left[u_{2 d}, u_{2 q}\right]^{T}, \boldsymbol{F}_{0}=\boldsymbol{F}_{00}+\Delta \boldsymbol{F}_{0}, \boldsymbol{G}=\boldsymbol{G}_{0}+\Delta \boldsymbol{G}, \boldsymbol{F}_{1}=\boldsymbol{F}_{10}+\Delta \boldsymbol{F}_{1}$, $\boldsymbol{F}_{20}=\boldsymbol{F}_{20}+\Delta \boldsymbol{F}_{2}$

$$
\begin{aligned}
& \boldsymbol{F}_{00}=-\left[\begin{array}{cc}
T_{10} & 0 \\
0 & T_{10}
\end{array}\right], \boldsymbol{G}_{0}=\left[\begin{array}{cc}
T_{20} & 0 \\
0 & T_{20}
\end{array}\right], \boldsymbol{F}_{10}=\left[\begin{array}{cc}
0 & \alpha_{10} \\
\alpha_{40} & 0
\end{array}\right], \boldsymbol{F}_{20}=\left[\begin{array}{cc}
\alpha_{20} & \alpha_{50} \\
\alpha_{30} & \alpha_{60}
\end{array}\right], \\
& \Delta \boldsymbol{F}_{0}=-\left[\begin{array}{cc}
\Delta T_{1} & 0 \\
0 & \Delta T_{1}
\end{array}\right], \Delta \boldsymbol{G}=\left[\begin{array}{cc}
\Delta T_{2} & 0 \\
0 & \Delta T_{2}
\end{array}\right], \Delta \boldsymbol{F}_{1}=\left[\begin{array}{cc}
0 & \Delta \alpha_{1} \\
\Delta \alpha_{4} & 0
\end{array}\right], \Delta \boldsymbol{F}_{2}=\left[\begin{array}{cc}
\Delta \alpha_{2} & \Delta \alpha_{5} \\
\Delta \alpha_{3} & \Delta \alpha_{6}
\end{array}\right],
\end{aligned}
$$

and the uncertainties above are upper-bounded by

$$
\begin{gathered}
\left\|\Delta F_{0}\right\| \leq M_{F_{0}}, \quad\|\Delta G\| \leq M_{G}, \quad\left\|\Delta F_{1}\right\| \leq M_{F_{1}}, \quad\left\|\Delta F_{2}\right\| \leq M_{F_{2}} \\
\left\|\Delta u_{2 d q}\right\| \leq \sqrt{2} u_{2 e}, \quad\left\|\Delta i_{1 d q}\right\| \leq \sqrt{2} i_{1 e}, \quad\left\|\Delta i_{2 d q}\right\| \leq \sqrt{2} i_{2 e}
\end{gathered}
$$

where $M_{\Delta F_{0}}, M_{\Delta F_{0}}, M_{\Delta F_{0}}$, and $M_{\Delta F_{0}}$ are known positive constants; $\boldsymbol{u}_{2 e}$ is the rated voltage of the CW; $i_{1 e}$ is the rated current of PW; and $i_{2 e}$ is the rated current of the CW.

The inner-loop subsystem with the consideration of the influence of parameter variation can be analysed by

$$
\frac{\mathrm{d} i_{2 d q}}{\mathrm{~d} t}=\boldsymbol{F}_{00} i_{2 d q}+\left(\boldsymbol{G}_{0}+\Delta \boldsymbol{G}\right)\left(\boldsymbol{u}_{2 d q}+\boldsymbol{F}_{10} i_{2 d q}+\boldsymbol{F}_{20} \boldsymbol{i}_{1 d q}\right)+\boldsymbol{\rho}_{1}
$$

where the uncertainty $\rho_{0}(t)=\Delta \boldsymbol{F}_{0} \boldsymbol{i}_{2 d q}+\left(\boldsymbol{G}_{0}+\Delta \boldsymbol{G}\right)\left(\Delta \boldsymbol{F}_{1} \boldsymbol{i}_{2 d q}+\Delta \boldsymbol{F}_{2} \boldsymbol{i}_{1 d q}\right)$.

For dynamic decoupling, a feedforward compensation is introduced:

$$
u_{d q}=u_{2 d q}+F_{10} i_{2 d q}+F_{20} i_{1 d q} .
$$

Substituting (37) to (36), the inner-loop subsystem can berewritten as follows:

$$
\frac{\mathrm{d} i_{2 d q}}{\mathrm{~d} t}=F_{00} i_{2 d q}+G_{0} u_{d q}+\Delta G u_{d q}+\rho_{1}
$$

where $\rho_{1}(t)=\Delta G u_{d q}+\rho_{0}(t)$. The uncertainty and its derivative are bounded by $\left\|\rho_{1}(t)\right\| \leq M_{\rho_{1}}$, $\left\|\dot{\rho}_{1}(t)\right\| \leq M_{d \rho_{1}}$.

\section{Full-Order Terminal Sliding-Mode Controller Design}

Let $K_{u 0} \Delta \dot{I}_{2 r e f}=u_{0}$. A full-order terminal sliding manifold $s_{0} \in R^{1}$ is designed as

$$
s_{0}=\dot{e} \dot{U}+c_{0} e_{U}^{q / p}
$$

where $c_{0}$ is a constant, $q$ and $p$ are odds, and $0<q / p<1$.

Theorem 1. Based on the sliding manifold (39) and the virtual control law as follows, the outerloop voltage tracking error dynamics can be regulated to reach the presupposed manifold in finite time $t_{r 0} \leq\left(\left|s_{0}(0)\right| / \eta_{0}\right)$, thereafter remaining on it and converging to equilibrium point in finite time $t_{s 0} \leq p /\left[\left(c_{0}(p-q)\right)\left|e_{U}\left(t_{r 0}\right)\right|^{1-q / p}\right]$.

$$
\begin{gathered}
u_{0}=u_{0 e q}+u_{0 n} \\
u_{0 e q}=c_{0} e_{u}^{q / p}
\end{gathered}
$$




$$
\begin{gathered}
u_{0 n}=\int k_{0} \operatorname{sgn}\left(s_{0}\right) \mathrm{d} \tau \\
k_{0}=\frac{M_{\delta} M_{d e q}(t)+\eta_{0}}{1-M_{\delta}} \\
\Delta I_{2 r e f}=\int \frac{1}{K_{u 0}} c_{0} e_{U}^{q / p}+\frac{1}{K_{u 0}} \iint k_{0} \operatorname{sgn}\left(s_{0}\right) \mathrm{d} \tau \mathrm{d} \tau
\end{gathered}
$$

where $M_{\delta}$ is defined in (34), $M_{\text {deq }}(t)$ is the upper bound of $\dot{u}_{0 e q}$, and $\eta_{0}$ is a constant.

Proof of Theorem 1. According to (30), Equation (39) can be rewritten as

$$
\begin{aligned}
s_{0} & =\dot{e} \dot{U}+c_{0} e_{U}^{q / p} \\
& =-K_{u 0} \Delta \dot{I}_{2 r e f}-\Delta K_{u} \Delta \dot{I}_{2 r e f}+K_{u 0} \ddot{e}_{i d}+c_{0} e_{U}^{q / p} \\
& =-u_{0}-\delta_{U} u_{0}+K_{u 0} \ddot{e}_{i d}+c_{0} e_{U}^{q / p} .
\end{aligned}
$$

A Lyapunov function is defined as $V_{0}=0.5 s_{0}^{2}$, and the time derivative of $V_{0}$ is expressed as

$$
\begin{aligned}
\dot{V}_{0} & =s_{0} \dot{s}_{0}=-s_{0} \dot{u}_{0 n}-s_{0} \delta_{U} \dot{u}_{0}+s_{0} K_{u 0} \ddot{e}_{i d} \\
& =-s_{0} \dot{u}_{0 n}-s_{0} \delta_{U} \dot{u}_{0 n}-s_{0} \delta_{U} \dot{u}_{0 e q}+s_{0} K_{u 0} \ddot{e}_{i d}
\end{aligned}
$$

Substituting the integral-type switching law (42) into the above equation gives

$$
\begin{aligned}
\dot{V}_{0} & \leq-k_{0}\left|s_{0}\right|+k_{0}\left|s_{0}\right|\left|\delta_{U}\right|+\left|s_{0}\right|\left|\delta_{U}\right|\left|\dot{u}_{0 e q}\right|+s_{0} K_{u 0} \ddot{e}_{i d} \\
& \leq-k_{0}\left(1-M_{\delta}\right)+M_{\delta}\left|s_{0}\right|\left|\dot{u}_{0 e q}\right|+s_{0} K_{u 0} \ddot{e}_{i d} .
\end{aligned}
$$

Thus, $\dot{V}_{0}$ is satisfied:

$$
\dot{V}_{0} \leq-\left\|s_{0}\right\|\left[\left(1-M_{\delta}\right) k_{0}-M_{\delta} M_{d 0 e q}(t)\right],
$$

where $M_{\text {deq }}(t)$ is the upper bound of $\dot{u}_{e q}$. When $s_{0} \neq 0$, considering the switching gain (44) yields

$$
\dot{V}_{0}=s_{0} \dot{s}_{0} \leq-\eta_{0}\left|s_{0}\right|+s_{0} K_{u 0} \ddot{e}_{i d} .
$$

The inner loop tracking error $e_{i d}$ and its derivative converge to zero in finite time and can be proven in the following Theorem 3 . Then, we have

$$
\dot{V}_{0} \leq-\eta_{0}\left|s_{0}\right| \leq-\sqrt{2} \eta_{0} V_{0}^{1 / 2}<0 .
$$

According to the lemma of finite time convergence in [38], the above equation illustrates that the outer loop-error tracking error dynamics will reach the ideal sliding manifold within finite time under the designed virtual control law (40). It is maintained on the sliding mainfold $s_{0}=0$ thereafter. During the sliding motion, the error dynamics converge to zero in finite time. It is obvious that the virtual control law overcomes the ummatched uncertainties completely. This completes the proof.

Let $\boldsymbol{e}_{i}=\boldsymbol{i}_{\text {ref }}-\boldsymbol{i}=\left[i_{\text {dref }}-i_{d}, 0-i_{q}\right]^{T}$ and $\boldsymbol{G}_{0} \boldsymbol{u}_{2 d q}=\boldsymbol{u}_{1}$. With the subsystem (38), the error dynamics can be given as

$$
\dot{\boldsymbol{e}}_{i}=\dot{\boldsymbol{i}}_{r e f}-\boldsymbol{F}_{00} \boldsymbol{i}_{2 d q}-\boldsymbol{u}_{1}-\Delta G G_{0}^{-1} \boldsymbol{u}_{1}-\boldsymbol{\rho}_{1} .
$$

A full-order terminal sliding manifold $s_{1} \in R^{2}$ is designed as

$$
s_{1}=\dot{e}_{i}+C_{1} e_{i}^{q / p}
$$

where $C_{1}=\operatorname{diag}\left(c_{11}, c_{12}\right), c_{11}>0, c_{12}>0$, and $q$ and $p$ are odds and $0<q / p<1$. 
Theorem 2. Based on the designed sliding surface (52), the virtual control law (40), and the following actual control law, the inner-loop current tracking error dynamics can be regulated to reach the sliding surface $s_{1}(t)=0$ in finite time $t_{r 1} \leq\left(\left\|s_{1}(0)\right\| / \eta_{1}\right)$. Then, the inner-loop tracking error $e_{i}$ and its derivative can converge to zero along the ideal sliding surface in finite time $t_{s 1} \leq \max \left\{p /\left[\left(c_{11}(p-q)\right)\left|e_{i d}\left(t_{r 1 d}\right)\right|^{1-q / p}\right], p /\left[\left(c_{12}(p-q)\right)\left|e_{i q}\left(t_{r 1 q}\right)\right|^{1-q / p}\right]\right\}$.

$$
\begin{aligned}
& \boldsymbol{u}_{1}=\boldsymbol{u}_{1 e q}+\boldsymbol{u}_{1 n} \\
& \boldsymbol{u}_{1 e q}=-\boldsymbol{F}_{00} \boldsymbol{i}_{2 d q}+\frac{1}{K_{u 0}}\left[\begin{array}{c}
c_{0}\left(-K_{u 0} \Delta I_{2 r e f}\right)^{q / p}+\int k_{0} \operatorname{sgn}\left(s_{0}\right) d \tau \\
0
\end{array}\right] \\
& \boldsymbol{u}_{1 n}=\int k_{1} \operatorname{sgn}\left(s_{0}\right) d \tau \\
& k_{1}=\frac{M_{G} M_{d 1 e q}(t) / T_{20}+c_{0} q M_{\delta} M_{\Delta I 2 r e f}^{(q-p) / p} M_{d \Delta I 2 r e f} / p+M_{d \rho_{1}}+\eta_{1}}{1-M_{G} / T_{20}} \\
& \boldsymbol{u}_{2 d q}=\boldsymbol{G}_{0}^{-1}\left(-\boldsymbol{F}_{00} \boldsymbol{i}_{2 d q}+\int k_{1} \operatorname{sgn}\left(\boldsymbol{s}_{0}\right) d \tau\right. \\
& \left.+\frac{1}{K_{u 0}}\left[\begin{array}{c}
c_{0}\left(-K_{u 0} \Delta I_{2 r e f}\right)^{q / p}+\int k_{0} \operatorname{sgn}\left(s_{0}\right) d \tau \\
0
\end{array}\right]\right)
\end{aligned}
$$

Proof of Theorem 2. According to (51), the sliding manifold (52) can be rewritten as follows:

$$
s_{1}=\dot{i}_{r e f}-F_{00} i_{2 d q}-u_{1}-\Delta G G_{0}^{-1} u_{1}-\rho_{1}+C_{1} e_{i}^{q / p} .
$$

Combined with (40), we have the following:

$$
\boldsymbol{s}_{1}=\frac{1}{K_{u 0}}\left[\begin{array}{c}
c_{0} e_{u}^{q / p}+\int \underset{0}{k_{0} \operatorname{sgn}\left(s_{0}\right) d \tau} \\
0
\end{array}\right]-\boldsymbol{F}_{00} i_{2 d q}-\boldsymbol{u}_{1}-\Delta G G_{0}^{-1} \boldsymbol{u}_{1}-\boldsymbol{\rho}_{1}+\boldsymbol{C}_{1} \boldsymbol{e}_{i}^{q / p} .
$$

Considering (30), the above can be rewritten as follows:

$$
\begin{aligned}
\boldsymbol{s}_{1} & =\frac{1}{K_{u 0}}\left[\begin{array}{c}
c_{0}\left(-K_{u 0} \Delta I_{2 r e f}-\Delta K_{u} \Delta I_{2 r e f}\right)^{q / p}+\int k_{0} \operatorname{sgn}\left(s_{0}\right) d \tau \\
0
\end{array}\right] \\
& -\boldsymbol{F}_{00} \boldsymbol{i}_{2 d q}-\boldsymbol{u}_{1}-\Delta G G_{0}^{-1} \boldsymbol{u}_{1}-\boldsymbol{\rho}_{1}+\boldsymbol{C}_{1} \boldsymbol{e}_{i}^{q / p} .
\end{aligned}
$$

Substitute the actual control law (53) into the above, then we get

$$
\boldsymbol{s}_{1}=\frac{1}{K_{u 0}}\left[\begin{array}{c}
c_{0}\left(-\Delta K_{u} \Delta I_{2 r e f}\right)^{q / p} \\
0
\end{array}\right]-\boldsymbol{u}_{1 n}-\Delta G G_{0}^{-1} \boldsymbol{u}_{1}-\boldsymbol{\rho}_{1} .
$$

Define a Lyapunov functionis as $V_{1}=0.5 s_{1}^{T} s_{1}$, and the derivative of $V_{1}$ is expressed as

$$
\begin{aligned}
\dot{V}_{1} & =\boldsymbol{s}_{1}^{T} \dot{\boldsymbol{s}}_{1} \\
& =-\boldsymbol{s}_{1}^{T} \boldsymbol{u}_{1 n}-\boldsymbol{s}_{1}^{T} \Delta G G_{0}^{-1} \dot{\boldsymbol{u}}_{1}-\boldsymbol{s}_{1}^{T} \dot{\boldsymbol{\rho}}_{1}+\frac{1}{K_{u 0}}\left[\begin{array}{c}
\left.c_{0}(q / p)\left(-\Delta K_{u} \Delta I_{2 r e f}\right)^{(q-p) / p} \dot{\Delta I_{2 r e f}}\right] . \\
0
\end{array} .\right.
\end{aligned}
$$

Substituting (53) into the above gives

$$
\begin{aligned}
\dot{V}_{1} & =\frac{1}{K_{u 0}}\left[\begin{array}{c}
c_{0}(q / p)\left(-\Delta K_{u} \Delta I_{2 r e f}\right)^{(q-p) / p} \Delta \dot{I}_{2 r e f} \\
0
\end{array}\right] \\
& -\boldsymbol{s}_{1}^{T} \boldsymbol{u}_{1 n}-s_{1}^{T} \Delta G G_{0}^{-1} \dot{\boldsymbol{u}}_{1 n}-\boldsymbol{s}_{1}^{T} \Delta G \boldsymbol{G}_{0}^{-1} \dot{\boldsymbol{u}}_{1 e q}-\boldsymbol{s}_{1}^{T} \dot{\boldsymbol{\rho}}_{1} .
\end{aligned}
$$


Thus,

$$
\begin{aligned}
\dot{V}_{1} & \leq\left\|\boldsymbol{s}_{1}\right\|\left(-k_{1}+k_{1}\|\Delta \boldsymbol{G}\|\left\|\boldsymbol{G}_{0}^{-1}\right\|+\|\Delta \boldsymbol{G}\|\left\|\boldsymbol{G}_{0}^{-1}\right\|\left\|\dot{\boldsymbol{u}}_{1 e q}\right\|\right. \\
& \left.+\frac{c_{0} q}{p}\left\|\delta_{U}\right\|\left\|\Delta I_{2 r e f}^{(q-p) / p}\right\|\left\|\Delta \dot{I}_{2 r e f}\right\|+\left\|\dot{\boldsymbol{\rho}}_{1}\right\|\right) .
\end{aligned}
$$

From the above, the upper bound of the actual control law can be estimated as

$$
\left\|\dot{\boldsymbol{u}}_{1}\right\| \leq\left\|\dot{\boldsymbol{u}}_{1 e q}\right\|+\left\|\dot{\boldsymbol{u}}_{1 n}\right\| \leq \sqrt{2} k_{1}+M_{d 1 e q}(t) .
$$

According to (44), the upper bound of $\Delta I_{2 r e f}$ and its derivative are estimated as

$$
\begin{array}{r}
\Delta I_{2 r e f}=\int \frac{1}{K_{u 0}} c_{0} e_{u}^{q / p}+\frac{1}{K_{u 0}} \iint k_{0} \operatorname{sgn}\left(s_{0}\right) d \tau \leq M_{\Delta I 2 r e f} \\
\Delta \dot{I}_{2 r e f}=\frac{1}{K_{u 0}} c_{0} e_{u}^{q / p}+\frac{1}{K_{u 0}} \int k_{0} \operatorname{sgn}\left(s_{0}\right) d \tau \leq M_{d \Delta I 2 r e f} .
\end{array}
$$

Combined with adaptive switching gain (56), when $V_{1} \neq 0$

$$
\begin{aligned}
\dot{V}_{1} & \leq\left\|s_{1}\right\|\left[\begin{array}{c}
-k_{1}\left(1-M_{G} / T_{20}\right)+M_{G} M_{d 1 e q}(t) / T_{20} \\
+c_{0} q M_{\delta} M_{\Delta I 2 r e f}^{(q-p) / p} M_{d \Delta I 2 r e f} / p+M_{d \rho_{1}}
\end{array}\right] \\
& \leq-\sqrt{2} \eta_{1} V_{1}^{1 / 2} .
\end{aligned}
$$

Similar to the proof of Theorem 1, the above equation means that the error dynamic approaches the ideal sliding surface $s_{1}=0$ in finite time. Additionally, the tracking errors of $\mathrm{CW}$ current and its derivative converge to the equilibrium point in finite time. From Theorem $1, e_{U}$ can converge to zero in finite time. This complete the proof.

According to Theroems 1 and 2, the PW voltage controller is designed for the outerloop and the CW current vector controllers are utilized for the inner-loop. The block diagram of the control scheme of the BDFIG as shown in Figure 2. The phase-locked loop (PLL) is used to track the amplitude of the output voltage, which is omitted here for space due to its simplicity.

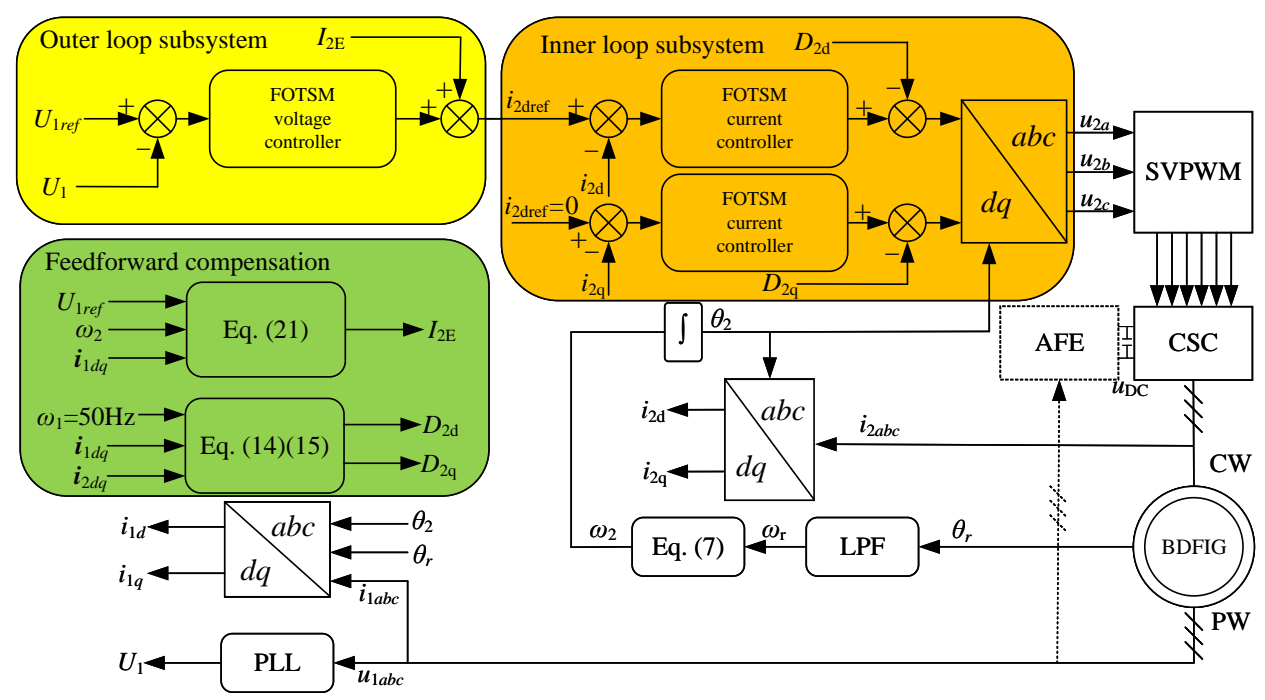

Figure 2. Block diagram of the control scheme of the standalone BDFIG. 


\section{Simulations}

In order to demonstrate the effectiveness of the proposed method, PI controllers, linear sliding mode (LSM), and FOTSM controllers are designed for performance comparison. The parameters of the BDFIG are listed in Table 1, and the controllers design parameters in Table 2.

Table 1. The parameters of the BDFIG.

\begin{tabular}{ccc}
\hline Symbol & Mean & Value \\
\hline$S_{e}$ & Capacity & $30 \mathrm{kVA}$ \\
$\omega_{e}$ & Speed range & $600-1200 \mathrm{rpm}$ \\
$p_{1}, p_{2}$ & PW and CW pole pairs & 1,3 \\
$U_{1 e}, I_{1 e}$ & PW rate voltage and current & $380 \mathrm{~V}, 45 \mathrm{~A}$ \\
$U_{2 e}, I_{2 e}$ & CW rate voltage and current & $0-350 \mathrm{~V}, 0-50 \mathrm{~A}$ \\
$R_{1}$ & PW resistances & $0.4034 \Omega$ \\
$R_{2}$ & CW resistances & $0.2608 \Omega$ \\
$R_{r}$ & Rotor resistances & $0.3339 \Omega$ \\
$L_{1}$ & PW inductances & $474.9 \mathrm{mH}$ \\
$L_{2}$ & CW inductances & $32.16 \mathrm{mH}$ \\
$L_{r}$ & rotor inductances & $225.2 \mathrm{mH}$ \\
$L_{1 r}$ & Mutual inductance between PW and rotor & $306.9 \mathrm{mH}$ \\
$L_{2 r}$ & Mutual inductance between CW and rotor & $25.84 \mathrm{mH}$ \\
\hline
\end{tabular}

Table 2. Controller design parameters.

\begin{tabular}{ccc}
\hline Control & Outer Loop Controller Parameters & Inner Loop Controller Parameters \\
\hline PI & $K_{p}=0.07, K_{i}=18$ & $K_{p}=21.5, K_{i}=972$ \\
LSM & $c=300, k=1000$ & $K_{p}=21.5, K_{i}=972$ \\
FOTSM & $c_{0}=300, k_{0}=4000, q / p=3 / 5$ & $c_{11}=c_{12}=1000, k_{1}=6000, q / p=3 / 5$ \\
\hline
\end{tabular}

\subsection{Start-Up Response}

The BDFIG starts at $0 \mathrm{~s}$ and runs for $1.0 \mathrm{~s}$ in total. The speed of the prime mover is set as $700 \mathrm{rpm}$ in the simulations. In order to characterize the parameter mismatch caused by the change in temperature and frequency, the resistances and inductances are set as $105 \%$ of the real values in the simulations. The reference frequency and amplitude of the $\mathrm{PW}$ voltage are $50 \mathrm{~Hz}$ and $327 \mathrm{~V}$, respectively. The load is a balance three-phase resistive load with resistances of $25 \Omega$ for each phase. The PI, LSM, and the proposed FOTSM controllers during the voltage start-up process are shown in Figure 3. It can be seen that the settling time of the voltage response under PI, LSM, and FOTSM are $0.079 \mathrm{~s}, 0.074 \mathrm{~s}$, and $0.028 \mathrm{~s}$, respectively. The BDFIG under the proposed FOTSM controller has a better dynamic response. It is obvious that the accuracy of the voltage response is enhanced and that the ripple is reduced. Figure 4 shows that the d-axis current response under the FOTSM can approach the given current faster than the PI and the LSM. It is evident that the ripple of $q$-axis current under the FOTSM is smaller than PI and LSM in Figure 5 owing to the integral-type control law and the adaptive switching gain. In Figure 6, the frequency under the PI and the LSM has a larger ripple than that under the FOTSM. 


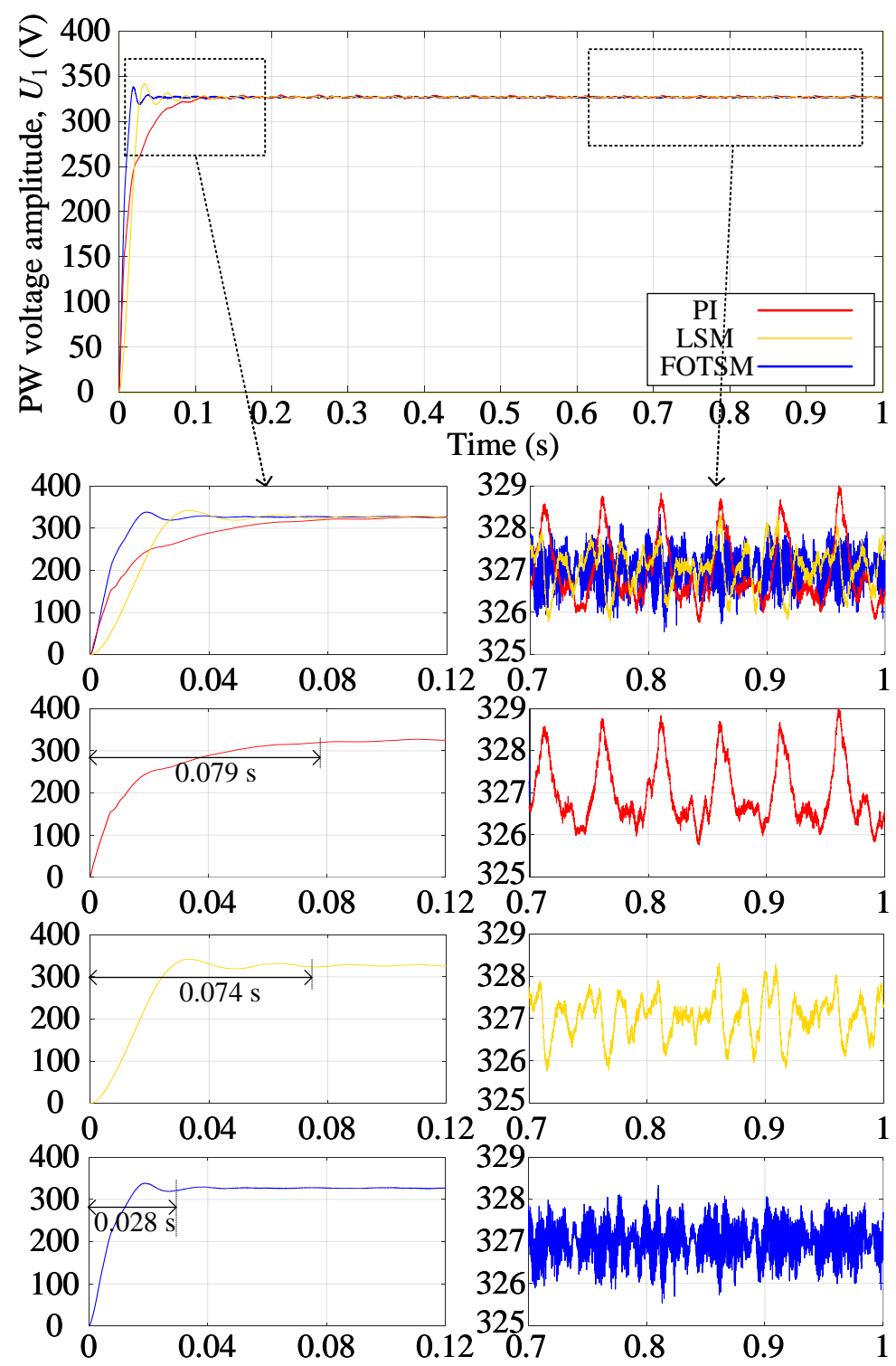

Figure 3. The PW-voltage amplitude responses under PI, LSM, and FOTSM during the start-up process.

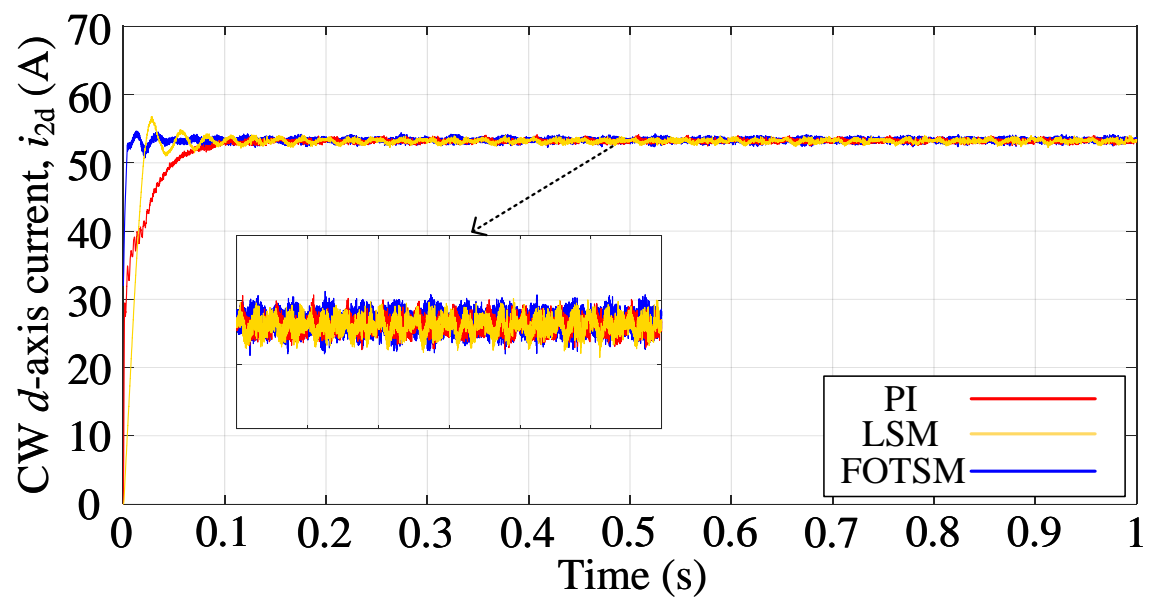

Figure 4. The CW $d$-axis current responses under PI, LSM, and FOTSM during the start-up process. 


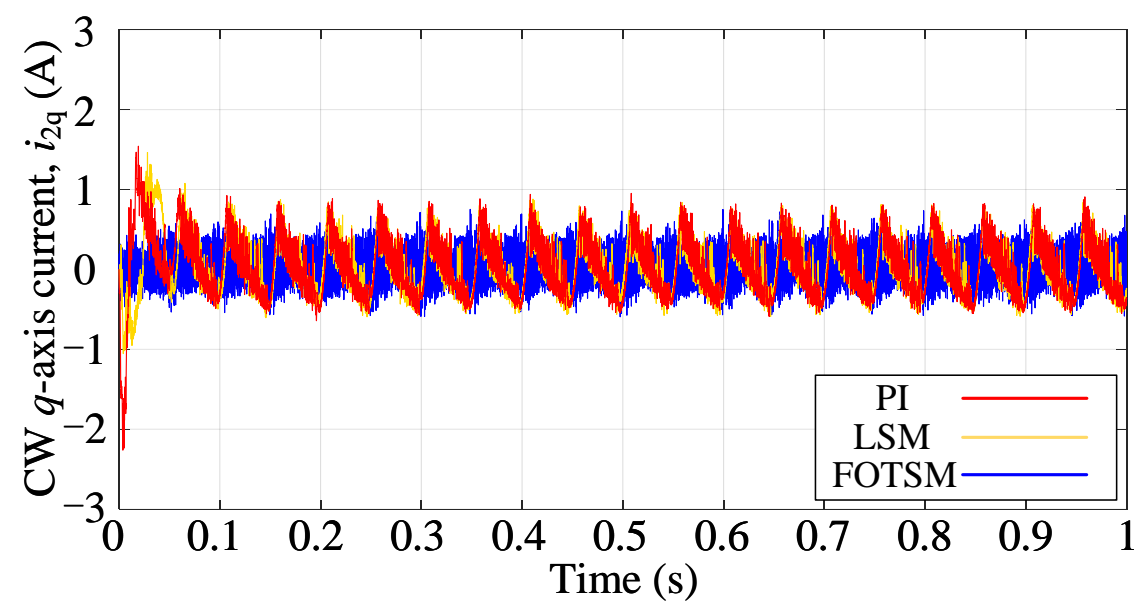

Figure 5. The CW q-axis current responses under PI, LSM, and FOTSM during the start-up process.

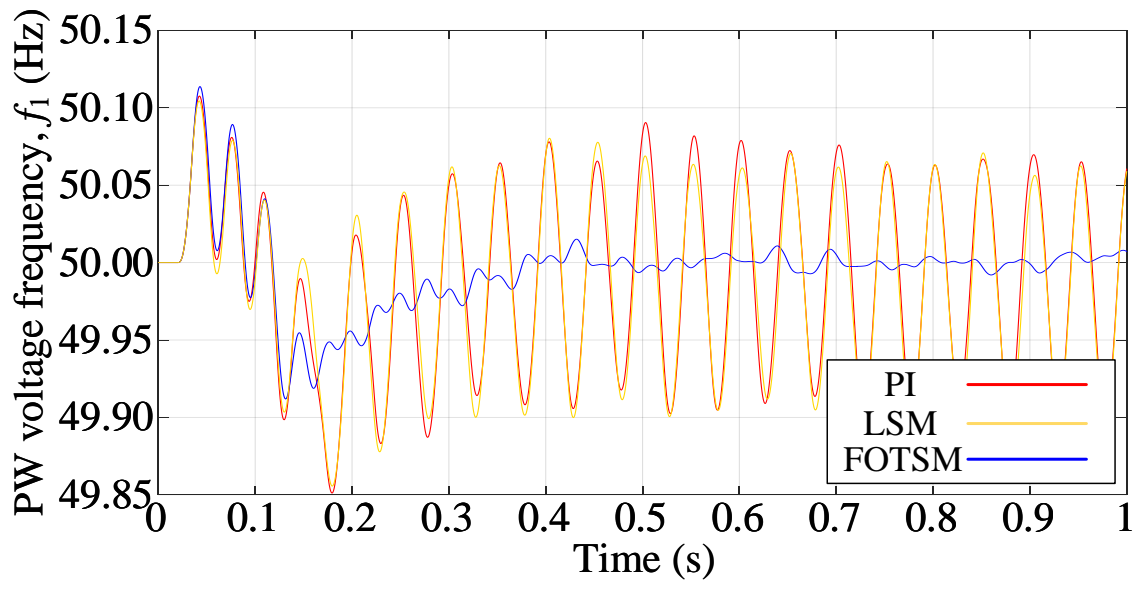

Figure 6. The PW voltage frequency responses under PI, LSM, and FOTSM during the start-up process.

\subsection{Load Adding Response}

In order to attest to the robustness of the three control methods, another balance three-phase resistive load with resistances of $120 \Omega$ for each phase is added to the system in $0.5 \mathrm{~s}$. The condition of the simulations is the same as the start-up response and the simulation results are shown in Figures 7-10. According to the simulation results shown in Figure 8 that the voltage amplitude drops under the PI, the LSM and the FOTSM are $21 \mathrm{~V}$, $22 \mathrm{~V}$, and $16 \mathrm{~V}$, respectively. In addition, the settling time of the voltage response under the $\mathrm{PI}$, the LSM, and the FOTSM are $0.045 \mathrm{~s}, 0.042 \mathrm{~s}$, and $0.008 \mathrm{~s}$, respectively, which means that the proposed FOTSM has a faster dynamic response and stronger robustness. It is obvious that the tracking error of the PW voltage amplitude under FOTSM is still smaller than the PI and the LSM when the load is added. In Figure 8, the current response under the FOTSM can converge to the equilibrium point faster than the PI and the LSM when the load is added. It can be seen from Figure 9 that the ripple of the $q$-axis current under the FOTSM is smaller than those under the PI and the LSM. In Figure 10, the fluctuation of frequency under the PI, the LSM, and the FOTSM is similar when the balance three-phase resistive load is added. However, the FOTSM has a faster recovery time and smaller ripple in steady-state than that under the PI and the LSM. These results further verify that the proposed FOTSM has a better robustness against the uncertainties. 


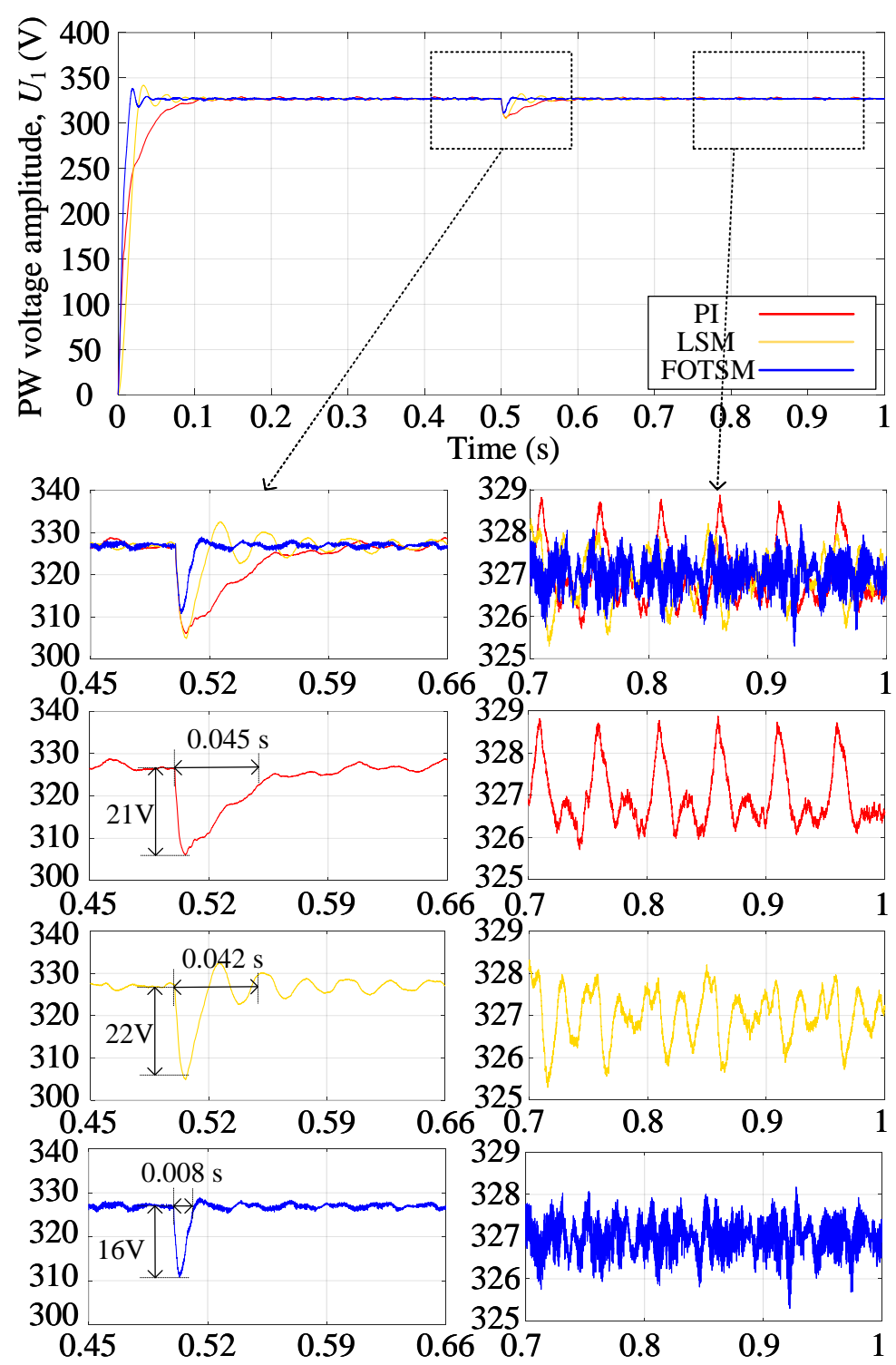

Figure 7. The PW-voltage amplitude responses under PI, LSM, and FOTSM during the load adding process.

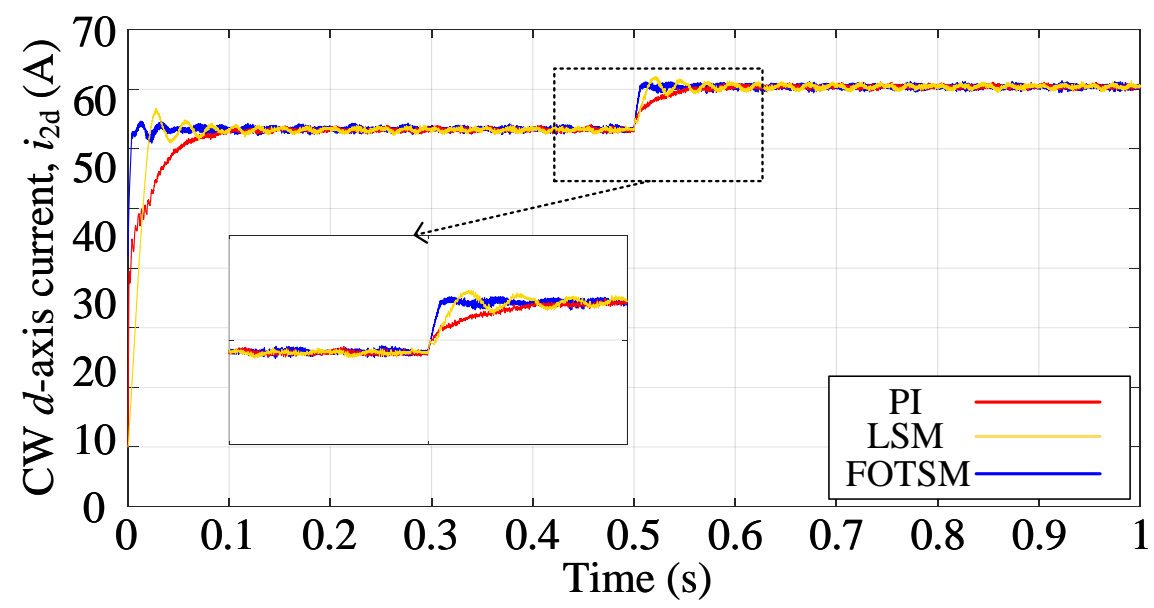

Figure 8. The CW $d$-axis current responses under PI, LSM, and FOTSM during the load adding process. 


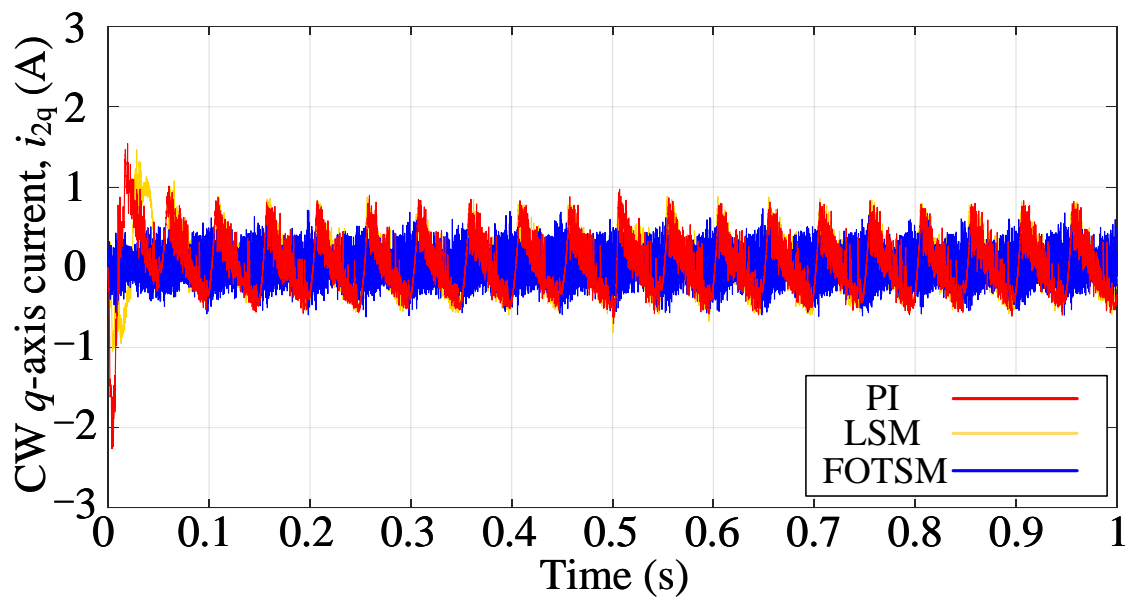

Figure 9. The CW $q$-axis current responses under PI, LSM, and FOTSM during the load adding process.

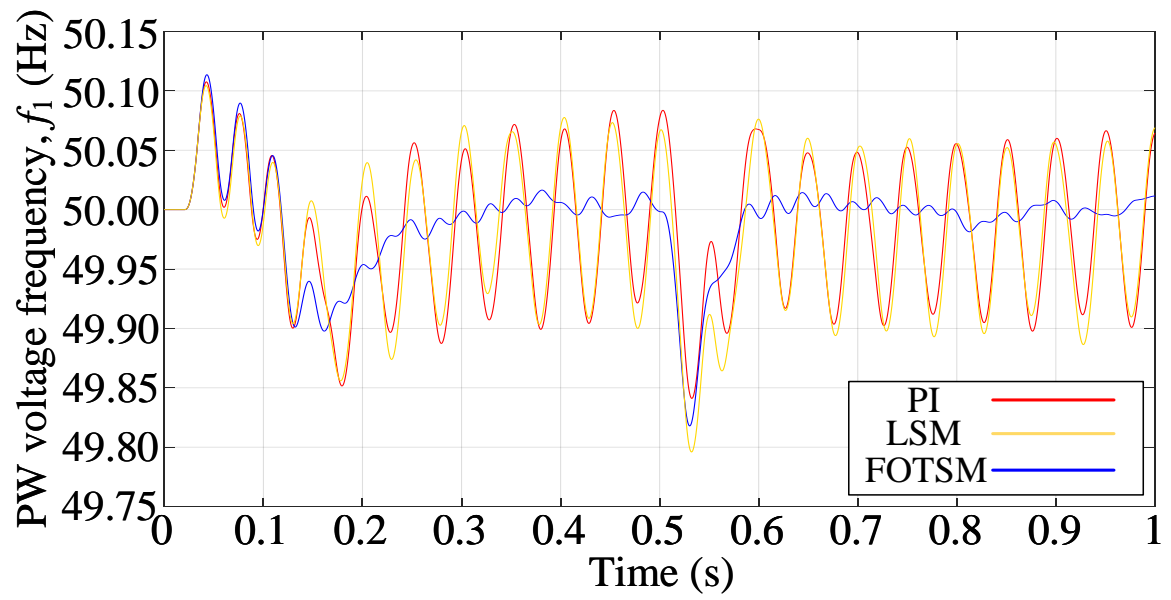

Figure 10. The PW voltage frequency responses under PI, LSM, and FOTSM during the load adding process.

\subsection{Voltage Change Response}

In order to demonstrate the dynamic response of the BDFIG using the FOTSM controllers. The reference of output PW-voltage amplitude is changed from $327 \mathrm{~V}$ to $360 \mathrm{~V}$ at $0.5 \mathrm{~s}$. The condition of the simulations is the same as the start-up response and the simulation results are shown in Figures 11-14. It can be concluded from Figure 11 that the settling time of the voltage response under the PI, the LSM, and the FOTSM are $0.045 \mathrm{~s}$, $0.035 \mathrm{~s}$, and $0.006 \mathrm{~s}$, respectively, when the reference of voltage amplitude is changed from $327 \mathrm{~V}$ to $360 \mathrm{~V}$ at $0.5 \mathrm{~s}$. Meanwhile, the precision of the output voltage under the FOTSM is better than those under the PI and the LSM. Figure 12 shows the $d$-axis current response under PI-, LSM-, and FOTSM- controllers, which verifies that the proposed method has a fast dynamic response. It is obvious from Figure 13 that the $q$-axis current response has a smaller ripple than those under the PI and the LSM. In Figure 14, the frequency of PW voltage response under the FOTSM has better precision and smaller fluctuation than the PI and the LSM in the condition of voltage change. Therefore, it can be concluded that the proposed FOTSM controllers have advantages in dynamic response and robustness against the uncertainties caused by parameter perturbation. 


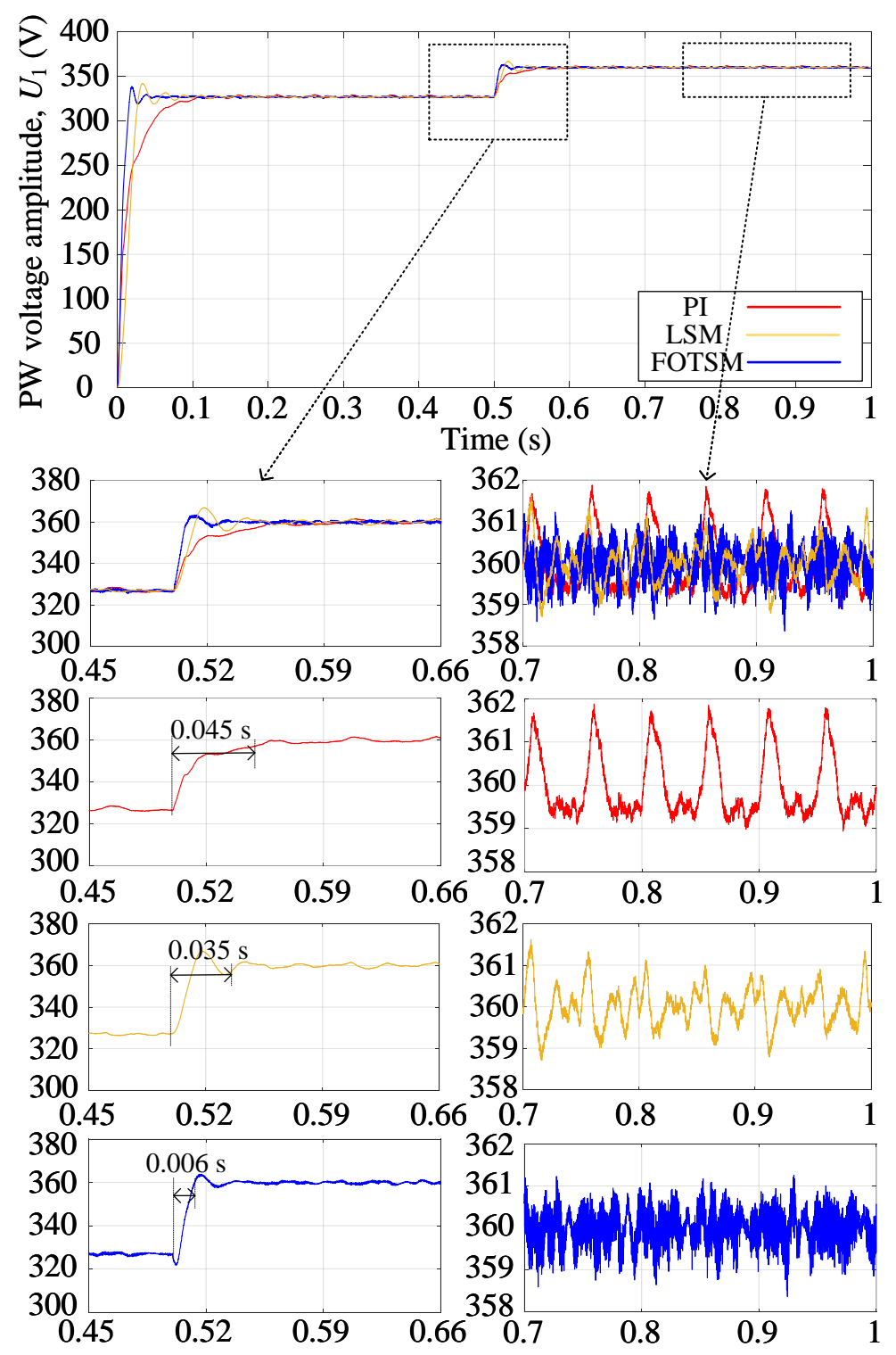

Figure 11. The PW-voltage amplitude responses under PI, LSM, and FOTSM during the voltage change process.

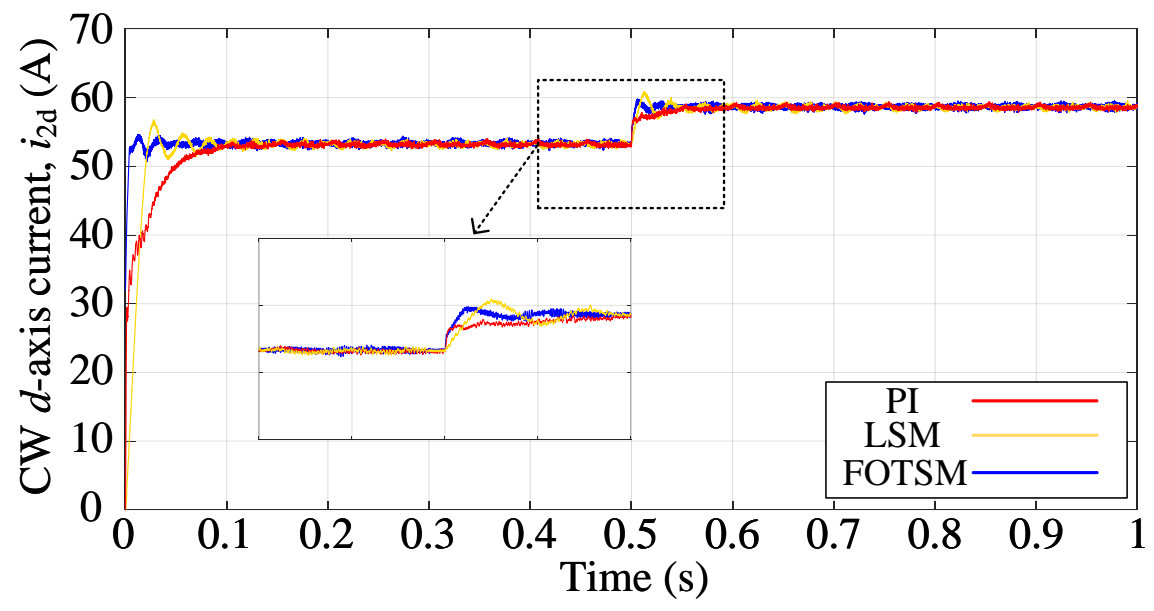

Figure 12. The CW $d$-axis current responses under PI, LSM, and FOTSM during the voltage change process. 


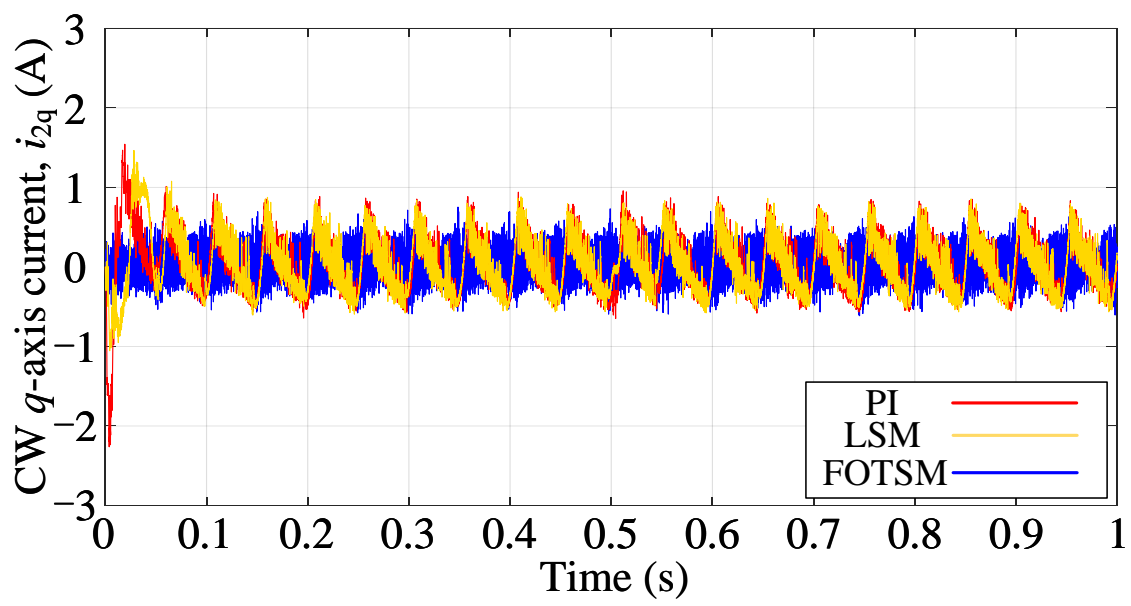

Figure 13. The CW $q$-axis current responses under PI, LSM, and FOTSM during the voltage change process.

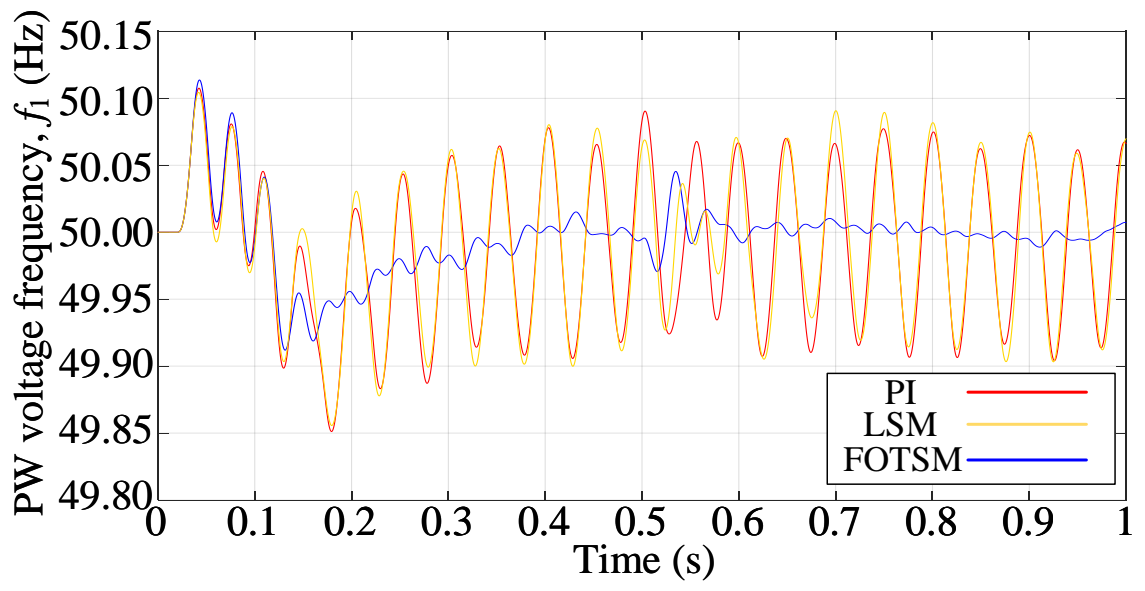

Figure 14. The PW voltage frequency responses under PI, LSM, and FOTSM during the voltage change process.

\section{Discussion}

For the implementation of high-performance BDFIG-based islanded microgrids, a sensorless control method, a harmonic elimination method, an adaptive gain to reduce the complexity of the control gain design, and an experimental study of the proposed control method will be our future work.

\section{Conclusions}

In this paper, a novel FOTSM control method was proposed to enhance the accuracy, the rapidness, and the robustness of the BDFIG-based islanded microgrids and to improve the output voltage quality. Different from the existing control methods, all of the matched and mismatched uncertainties in the BDFIG system are fully considered. With the use of the virtual control technique-based FOTSM, the tracking errors can be forced to converge to zero in finite time, and the matched and mismatched uncertainties can be thoroughly compensated, which improve the disturbance rejection capability of the BDFIG system. Owing to the integral control law with the adaptive gain, the output signals of the proposed controllers are smooth. Comprehensive simulation results have proved the effectiveness and feasibility of the proposed method in this work. 
Author Contributions: M.Z.: conceptualization, algorithm innovation, methodology, and writingoriginal draft; H.S.: data and formal analysis, investigation, software, simulation, and writingoriginal draft; Y.L.: conceptualization, simulation, investigation, methodology, writing-original draft; W.C.: investigation, and writing-review and editing; W.X.: formal analysis, and writingreview and editing; D.W.: data and formal analysis, software, and simulation. All authors have read and agreed to the published version of the manuscript.

Funding: This work was supported in part by the Excellent Youth Fund of Shandong Natural Science Foundation under grant ZR2020YQ40; by the fund from the Science, Technology, and Innovation Commission of Shenzhen Municipality under grant JCYJ20190809101205546; by the Fundamental Research Funds for the Central Universities of China under grant 2021XXJS002; by Heilongjiang Postdoctoral Financial Assistance (LBHZ19166); by the Heilongjiang Industrial Revitalization Major Project on Engineering and Science (2019ZX02A01); and by the National Natural Science Foundation of China (NSFC) (62073095).

Institutional Review Board Statement: Not applicable.

Informed Consent Statement: Not applicable.

Data Availability Statement: Data sharing not applicable.

Acknowledgments: This research was funded by the New Energy Motor System and Key Materials Innovation Center at Harbin University of Science and Technology and the Center for Energy Conversion System at Huazhong University of Science and Technology.

Conflicts of Interest: The authors declare no conflict of interest. The funders had no role in the design of the study; in the collection, analyzes, or interpretation of data; in the writing of the manuscript; or in the decision to publish the results.

\author{
Abbreviations \\ The following abbreviations are used in this manuscript: \\ BDFIG Brushless doubly fed induction generator \\ LSM Linear sliding-mode \\ FOTSM Full-order terminal sliding-mode \\ DFIG Doubly fed induction generator \\ IM Induction motor \\ PW Power winding \\ CW Control winding \\ VC Vertor control \\ DTC Direct torque control \\ MIMO Mutiple-input-mutiple-output \\ PI Practial proportional-integral \\ CSC CW side converter \\ AFT Active front end \\ MRAC Model reference adaptive control \\ MPC Model predictive control \\ ADRC Active disturbance rejection control \\ SMC Sliding-mode control \\ SVPWM Signals of space vector pulse width modulationn \\ PWM Pulse width modulation \\ Nomenclature \\ $p_{1}, p_{2} \quad$ Pole pair numbers of PW and CW. \\ $\omega_{1}, \omega_{2} \quad$ Angular frequencies of PW and CW. \\ $\omega_{r}, \omega_{N} \quad$ Actual and natural synchronous rotor speeds. \\ $\theta_{2} \quad$ Angular position of CW current vectors. \\ $R_{1}, R_{2}, R_{r} \quad$ Resistances of PW, CW, and rotor. \\ $L_{1}, L_{2}, L_{r} \quad$ Self-inductances of PW, CW, and rotor. \\ $L_{1 r} \quad$ Mutual inductance between PW and rotor. \\ $L_{2 r} \quad$ Mutual inductance between $\mathrm{CW}$ and rotor.
}




$\begin{array}{ll}i_{1}, i_{2}, i_{r} & \text { Currents of PW, CW, and rotor. } \\ u_{1}, u_{2}, u_{r} & \text { Voltages of PW, CW, and rotor. } \\ \phi_{1}, \phi_{2}, \phi_{r} & \text { Fluxes of PW, CW, and rotor. } \\ s & \text { Differential operator, } \mathrm{d} / \mathrm{d} t . \\ u & \text { Voltage amplitude. } \\ \boldsymbol{u} & \text { Voltage vector. } \\ \boldsymbol{i} & \text { Current vector. } \\ r e f & \text { Reference value. } \\ a, b, c & \text { States in } a b c \text {-axis. } \\ d, q & \text { States in } d q \text {-axis. }\end{array}$

\section{References}

1. Zeng, Y.; Cheng, M.; Wei, X.; Zhang, G. Grid-Connected and Standalone Control for Dual-Stator Brushless Doubly Fed Induction Generator. IEEE Trans. Ind. Electron. 2021, 10, 9196-9206. [CrossRef]

2. Xu, W.; Hussien, M.; Liu, Y.; Islam, M.; Allam, S. Sensorless Voltage Control Schemes for Brushless Doubly-Fed Induction Generators in Stand-Alone and Grid-Connected Applications. IEEE Trans. Energy Convers. 2020, 35, 1781-1795. [CrossRef]

3. Sami, I.; Ullah, S.; Ali, Z.; Ullah, N.; Ro, J.-S. A Super Twisting Fractional Order Terminal Sliding Mode Control for DFIG-Based Wind Energy Conversion System. Energies 2020, 13, 2158. [CrossRef]

4. Min, L.; Chen, Y.; Debin, Z.; Jingyuan, S.; Yong, K. Virtual Synchronous Control Based on Control Winding Orientation for Brushless Doubly Fed Induction Generator (BDFIG) Wind Turbines Under Symmetrical Grid Faults. Energies 2019, $12,319$.

5. Taluo, T.; Ristić, L.; Jovanović, M. Dynamic Modeling and Control of BDFRG under Unbalanced Grid Conditions. Energies 2021, 14, 4297. [CrossRef]

6. Xu, W.; Elbabo M.; Omer M.; Liu, Y.; Islam, M.R. Negative Sequence Voltage Compensating for Unbalanced Standalone Brushless Doubly-Fed Induction Generator. IEEE Trans. Power Electron. 2020, 35, 667-680. [CrossRef]

7. Ullah, N.; Sami, I.; Chowdhury, M.; Techato, K.; Alkhammash, H. Artificial Intelligence Integrated Fractional Order Control of Doubly Fed Induction Generator-Based Wind Energy System. IEEE Access 2021, 9, 5734-5748. [CrossRef]

8. Han, P.; Cheng, M.; Jiang, Y.l.; Chen, Z. Torque/Power Density Optimization of a Dual-Stator Brushless Doubly-Fed Induction Generator for Wind Power Application. IEEE Trans. Ind. Electron. 2017, 64, 9864-9875. [CrossRef]

9. Barati, F.; McMahon, R.; Shao, S.Y.; Abdi, E.; Oraee, H. Generalized Vector Control for Brushless Doubly Fed Machines with Nested-Loop Rotor. IEEE Trans. Ind. Electron. 2013, 60, 2477-2485. [CrossRef]

10. Izaskun S.; Javier P.; Miguel A.R.; Gonzalo A. Direct torque control design and experimental evaluation for the brushless doubly fed machine. Energy Convers. Manag. 2011, 52, 142-149.

11. Shao, S.Y.; Abdi, E.; McMahon, R. Low-Cost Variable Speed Drive Based on a Brushless Doubly-Fed Motor and a Fractional Unidirectional Converter. IEEE Trans. Ind. Electron. 2008, 59, 317-325. [CrossRef]

12. Zhang, A.; Wang, X.; Jia, W.X.; Ma, Y. Indirect Stator-Quantities Control for the Brushless Doubly Fed Induction Machine. IEEE Trans. Power Electron. 2014, 29, 1392-1401. [CrossRef]

13. Sebtahmadi, S.S.; Pirasteh, H.; Aghay Kaboli, S.H.; Radan, A.; Mekhilef, S. A 12-Sector Space Vector Switching Scheme for Performance Improvement of Matrix-Converter-Based DTC of IM Drive. IEEE Trans. Power Electron. 2015, 30, 3804-3817. [CrossRef]

14. Liu, Y.; Xu, W.; Zhu, J.G.; Blaabjerg, F. Sensorless Control of Standalone Brushless Doubly Fed Induction Generator Feeding Unbalanced Loads in a Ship Shaft Power Generation System. IEEE Trans. Ind. Electron. 2019, 66, 739-749. [CrossRef]

15. Lu, X.; Lai, J.; Liu, G. Master-Slave Cooperation for Multi-DC-MGs via Variable Cyber Networks. IEEE Trans. Cybern. 2021. [CrossRef]

16. Vargas, U.; Ramirez, A. Extended Harmonic Domain Model of a Wind Turbine Generator for Harmonic Transient Analysis. IEEE Trans. Power Deliv. 2016, 31, 1360-1368. [CrossRef]

17. Kim, J.; Seok, J.K.; Muljadi, E.; Kang, Y. Adaptive $Q-V$ Scheme for the Voltage Control of a DFIG-Based Wind Power Plant. IEEE Trans. Power Electron. 2016, 31, 3586-3599. [CrossRef]

18. Qin, B.; Sun, H.; Ma, J.; Li, W.; Ding, T.; Wang, Z.; Zomaya, A.Y. Robust $H \infty$ Control of Doubly Fed Wind Generator via State-Dependent Riccati Equation Technique. IEEE Trans. Power Syst. 2021, 34, 2390-2400. [CrossRef]

19. Sharmila, V.; Rakkiyappan, R.; Joo, Y.H. Fuzzy Sampled-Data Control for DFIG-Based Wind Turbine with Stochastic Actuator Failures. IEEE Trans. Syst. Man Cybern. Syst. 2021, 51, 2199-2211. [CrossRef]

20. Lu, L.; Avila, N.F.; Chu, C.C.; Yeh, T.W. Model Reference Adaptive Back-Electromotive-Force Estimators for Sensorless Control of Grid-Connected DFIGs. IEEE Trans. Ind. Appl. 2018, 54, 1701-1711. [CrossRef]

21. Errouissi, R.; Al-Durra, A.; Muyeen, S.M.; Leng, S.Y.; Blaabjerg, F. Offset-Free Direct Power Control of DFIG Under ContinuousTime Model Predictive Control. IEEE Trans. Power Electron. 2017, 32, 2265-2277. [CrossRef]

22. Tohidi, A.; Hajieghrary, H.; Hsieh, M.A. Adaptive Disturbance Rejection Control Scheme for DFIG-Based Wind Turbine: Theory and Experiments. IEEE Trans. Ind. Appl. 2016, 52, 2006-2015. [CrossRef]

23. Lu, X.; Lai, J. Communication Constraints for Distributed Secondary Control of Heterogenous Microgrids: A Survey. IEEE Trans. Ind. Appl. 2021. [CrossRef] 
24. Lai, J.; Lu, X.; Dong, Z.; Cheng, S. Resilient Distributed Multiagent Control for AC Microgrid Networks Subject to Disturbances. IEEE Trans. Syst. Man Cybern. Syst. 2021. [CrossRef]

25. Ruiz-Cruz, R.; Sanchez, E.N.; Loukianov, A.G.; Ruz-Hernandez, J.A. Real-Time Neural Inverse Optimal Control for a Wind Generator. IEEE Trans. Sustain. Energy 2019, 10, 1172-1183. [CrossRef]

26. Hu, J.; He, N.; Hu, B.; He, Y.; Zhu, Z. Direct Active and Reactive Power Regulation of DFIG Using Sliding-Mode Control Approach. IEEE Trans. Energy Convers. 2010, 25, 1028-1039. [CrossRef]

27. Lascu, C.; Boldea, I.; Blaabjerg, F. Direct torque control of sensorless induction motor drives: A sliding-mode approach. IEEE Trans. Ind. Appl. 2004, 40, 582-590. [CrossRef]

28. Zhou, M.; Cheng, S.; Feng, Y.; Xu, W.; Wang, L.; Cai, W. Full-Order Terminal Sliding-Mode based Sensorless Control of Induction Motor with Gain Adaptation. IEEE J. Emerg. Sel. Top. Power Electron. 2021. [CrossRef]

29. Sun, D.; Wang, X.; Nian, H.; Zhu, Z. A Sliding-Mode Direct Power Control Strategy for DFIG Under Both Balanced and Unbalanced Grid Conditions Using Extended Active Power. IEEE Trans. Power Electron. 2018, 33, 1313-1322. [CrossRef]

30. Sadeghi, R.; Madani, S.M.; Ataei, M.; Agha Kashkooli, M.R.; Ademi, S. Super-Twisting Sliding Mode Direct Power Control of a Brushless Doubly Fed Induction Generator. IEEE Trans. Ind. Electron. 2018, 65, 9147-9156. [CrossRef]

31. Amin, I.K.; Uddin, M.N. Nonlinear Control Operation of DFIG-Based WECS Incorporated With Machine Loss Reduction Scheme. IEEE Trans. Power Electron. 2020, 35, 7031-7044. [CrossRef]

32. Chen, S.; Cheung, N.C.; Wong, K.C.; Wu, J. Integral Sliding-Mode Direct Torque Control of Doubly-Fed Induction Generators Under Unbalanced Grid Voltage. IEEE Trans. Energy Convers. 2010, 25, 356-368. [CrossRef]

33. Evangelista, C.; Valenciaga, F.; Puleston, P. Active and Reactive Power Control for Wind Turbine Based on a MIMO 2-Sliding Mode Algorithm with Variable Gains. IEEE Trans. Energy Convers. 2013, 28, 682-689. [CrossRef]

34. Evangelista, C.A.; Pisano, A.; Puleston, P.; Usai, E. Receding Horizon Adaptive Second-Order Sliding Mode Control for DoublyFed Induction Generator Based Wind Turbine. IEEE Trans. Control Syst. Technol. 2016, 25, 73-84. [CrossRef]

35. Yan, X.; Cheng, M.; Xu, L.; Zeng, Y. Dual-Objective Control Using an SMC-Based CW Current Controller for Cascaded Brushless Doubly Fed Induction Generator. IEEE Trans. Ind. Appl. 2020, 56, 7109-7120. [CrossRef]

36. Zhang, G.; Yang, J.; Sun, Y.; Su, M.; Tang, W.; Zhu, Q.; Wang, H. A Robust Control Scheme Based on ISMC for the Brushless Doubly Fed Induction Machine. IEEE Trans. Power Electron. 2018, 33, 3129-3140. [CrossRef]

37. Yu, X.; Feng, Y.; Man, Z. Terminal Sliding Mode Control-An Overview. IEEE Open J. Ind. Electron. Soc. 2021, 2, 36-52. [CrossRef]

38. Feng, Y.; Zhou, M.; Zheng, X.; Han, F.; Yu, X. Full-order terminal sliding-mode control of MIMO systems with unmatched uncertainties. J. Frankl. Inst. 2017, 1, 1-22. [CrossRef] 